\title{
TAVR improves survival in inoperable patients with severe aortic stenosis
}

Transcatheter aortic valve replacement (TAVR) has emerged as an efficacious strategy to treat symptomatic severe aortic stenosis in high-risk patients who are not suited for surgical valve replacement. A new study now demonstrates that TAVR is more effective in reducing mortality than standard therapy in these patients.

Investigators in the PARTNER trial randomly assigned 342 high-risk, inoperable patients with aortic stenosis to receive either TAVR or standard therapy. The clinical outcome of left ventricular (LV) dysfunction was defined as LV ejection fraction (LVEF) $<50 \%$.

Baseline LV dysfunction was present in approximately one-third of all patients, and did not affect 30-day or 1-year survival after TAVR. However, baseline LV dysfunction was associated with increased mortality at 1 year in patients receiving standard therapy (59.3\% versus $45.8 \%$ with normal LVEF; HR 1.71, 95\% CI
1.08-2.71; $P=0.02)$. LVEF improvement, defined as an increase in LVEF $\geq 10 \%$ at 30 days, was observed in $48.7 \%$ of TAVR patients and $30.4 \%$ of those receiving standard therapy $(P=0.08)$. In patients with LV dysfunction who received standard therapy, LVEF improvement conferred favourable 1-year, but not 2-year, survival.

"These data confirm the efficacy and safety of TAVR in inoperable patients with severe [aortic stenosis] and mild or moderate LV dysfunction," explain the investigators. "Future efforts should ... [clarify] the impact of more severe LV dysfunction and the lack of contractile reserve on outcomes of TAVR".

\section{Karina Huynh}

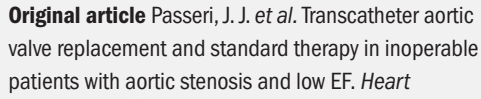

Original article Passeri, J. J. et al. Transcatheter aortic valve replacement and standard therapy in inoperable patients with aortic stenosis and low EF. Heart doi:10.1136/heartjnl-2014-306737 